\title{
INDEPENDENT SETS AND LACUNARITY FOR HYPERGROUPS
}

\author{
RICHARD C. VREM
}

(Received 20 May 1988; revised 25 June 1989)

Communicated by C. Sutherland

\begin{abstract}
Sets of independence are studied for compact abelian hypergroups and they are used, along with Riesz products, to investigate lacunarity questions on the dual object. It is shown that bounded Stechkin sets are always Sidon and that every bounded infinite subset of the dual contains an infinite Sidon set which is also a $\Lambda$ set. Independent sets are shown to always be Sidon and a necessary condition for Sidonicity is provided. A result of Pisier is used to show that for compact non-abelian groups Sidon and central $\Lambda$ are equivalent. Several applications are provided, primarily to questions regarding lacunarity on compact groups.
\end{abstract}

1980 Mathematics subject classification (Amer. Math. Soc.) (1985 Revision): 43 A 46, 43 A 40.

\section{Introduction and notation}

Lacunary sets have been studied extensively in a wide variety of settings. In this paper, we will consider lacunary sets within the framework of compact abelian hypergroups. Many of the results will be applied to draw conclusions about lacunarity and central lacunarity on compact nonabelian groups.

In Section 2, Riesz products are introduced for compact abelian hypergroups and some of their important properties are studied. The ideas and notation developed in Section 2 are used in Section 3 to define and compare various sets with independence properties. In particular, the familiar definitions of independence, quasi-independence, Rider and Stechkin sets

The author would like to thank the University of British Columbia Mathematics Department for its support and hospitality during his sabbatical leave in 1987.

(C) 1991 Australian Mathematical Society 0263-6115/91 \$A2.00+0.00 
are all extended to the setting of compact abelian hypergroups. Moreover, it is shown that every infinite subset of the dual contains an infinite quasiindependent set.

Sufficient conditions for a subset of the dual to be a Sidon set are presented in Section 4. It is shown that every bounded Stechkin set is a Sidon set and that every bounded infinite subset of the dual contains an infinite Sidon set which is also a $\Lambda$ set. An analogue of Kronecker's theorem is then used to show that independent sets are Sidon sets. A necessary condition for Sidonicity is provided in Section 3, in terms of the Fourier transform of point masses. A result of Pisier [16] is reformulated in Section 6 to show that for compact nonabelian groups a set is Sidon if and only if it is central $\Lambda$.

Applications of Sections 4 and 6 are provided in Section 7 within the context of lacunarity questions on compact nonabelian groups. Several questions posed by Parker [14] are discussed and the set of all compact connected groups with the property that every central Sidon set is also a Sidon set is shown to be exactly the set of all compact connected Lie groups. It is also shown that Hutchinson's result [8] that non-tall compact groups admit infinite Sidon sets is generalized by results in Section 4. Finally, in Section 8 the countable compact hypergroups introduced by Lasser [10] are used to illustrate several of the results presented in earlier sections.

We refer the reader to Jewett [9] for basic information about hypergroups (where they are called convos) and to [22] for specific details regarding lacunarity on compact (not necessarily abelian) hypergroups.

Unless otherwise noted, all the hypergroups $K$ under discussion in this paper will be assumed to be compact and abelian with dual objects $\widehat{K}$ which are also assumed to be hypergroups, that is,

$$
\sum_{\eta \in K^{-}} \delta_{\gamma^{*}} * \delta_{\psi}(\eta) \eta=\gamma \psi \text { for all } \gamma, \psi \in \widehat{K}
$$

If $(\widehat{\hat{K}}) \cong K$ we call $K$ strong. For each $x$ in $K, \delta_{x}$ will denote the point mass at $x$ and the map $x \rightarrow \check{x}$ will denote the involution on $K$ while the involution on $\widehat{K}$ is complex conjugation. If $A$ is a subset of any hypergroup we denote the subhypergroup generated by $A$ as $\langle A\rangle$. We denote Haar measure on $K$ by $m$.

Given a finite subset $P$ of $\widehat{K}$ the measure $\mu$ on $\widehat{K}$ formed by convolving all the point masses on $P$ will be denoted by $\mu=\prod_{\psi \in P} \delta_{\psi}$ and we write supp $\mu=\prod_{\psi \in P}\{\psi\}$. A subset $P$ of $K$ is called symmetric if $\bar{P} \equiv\{\bar{\psi}: \psi \epsilon$ $P\}=P$ and $P$ is called asymmetric if $1 \notin P$ (where 1 is the trivial character) and $\psi \in P$ with $\psi \neq \bar{\psi}$ implies $\bar{\psi} \notin P$. 
For $X \subseteq M(K)$ and $P \subseteq \widehat{K}$ let $X_{P}=\{\mu \in X: \widehat{\mu}(\psi)=0$ for all $\psi \notin P\}$. As in the case of groups, we call $P \subseteq \widehat{K}$ a Sidon set if $C_{P}(K) \subset A(K)$ where $A(K)$ denotes all those functions in $L^{1}(K)$ with absolutely convergent Fourier series. A list of conditions equivalent to Sidonicity are provided in [22, 2.2 and 2.5]. We call $P$ a $\Lambda$ set if there is a constant $k$ such that for all $q>2$ it follows that $\|f\|_{q} \leq k q^{1 / 2}\|f\|_{2}$ for all $f \in \operatorname{Trig}_{P}(K)$ where $\operatorname{Trig}(K)$ denotes all the trigonometric polynomials on $K$.

\section{Riesz products}

In this section we generalize the notion of Riesz products to compact abelian hypergroups. Also, the notation and machinery are developed to define various degrees of independence for hypergroups which will be presented in Section 3. The presentation in this section follows closely that found in Lopez and Ross [11].

Let $K$ be a compact abelian hypergroup with $P$ an asymmetric subset of $\widehat{K}$ and let $Q=P \cup \bar{P}$. Let $g$ be a hermitian function on $Q$ (that is, $g(\bar{\psi})=\overline{g(\psi)}$ for all $\psi$ in $Q$ ). For each finite symmetric subset $A$ of $Q$ define the Riesz product

$$
P_{A}=\prod_{\gamma \in A \cap P} r_{\gamma} \quad \text { where } r_{\gamma}=\left\{\begin{array}{l}
1+g(\gamma) \gamma+g(\bar{\gamma}) \bar{\gamma} \quad \text { if } \gamma \neq \bar{\gamma}, \\
1+g(\gamma) \gamma \quad \text { if } \gamma=\bar{\gamma} .
\end{array}\right.
$$

Note that $P_{A}$ is a real-valued function on $K$ and that the asymmetry of $P$ along with the symmetry of $A$ assure that each element of $A$ is accounted for precisely once in the Riesz product $P_{A}$.

We may rewrite the above Riesz product as

$$
P_{A}=\sum_{\gamma \in \widehat{K}} C_{A}(\gamma) \gamma
$$

where $C_{A}(\gamma)=\sum_{B}\left(\prod_{\psi \in B} g(\psi)\right)\left[\left(\prod_{\psi \in B} \delta_{\psi}\right)(\gamma)\right]$ and the sum ranges over all asymmetric subsets $B$ of $A$. Here we allow $B=\varnothing$ and the corresponding term in the sum is taken to be 1 . For $s \geq 0$ and $\gamma$ in $\widehat{K}$ define

$$
C_{A}^{s}(\gamma)=\sum_{B}\left(\prod_{\psi \in B} g(\psi)\right)\left[\left(\prod_{\psi \in B} \delta_{\psi}\right)(\gamma)\right]
$$

where the sum is over all asymmetric subsets $B$ of $A$ with $|B|=s$ (here $|B|$ denotes the cardinality of $B$ ). If there are no such sets $B$ we set $C_{A}^{s}(\gamma)=0$ and we define

$$
C_{A}^{0}(\gamma)= \begin{cases}1 & \text { if } \gamma=1 \\ 0 & \text { otherwise }\end{cases}
$$


Thus

$$
C_{A}(\gamma)=\sum_{s=0}^{|A|} C_{A}^{s}(\gamma)
$$

for each $\gamma$ in $\widehat{K}$. For any subset $N$ of $\widehat{K}$ not containing 1 and $s \geq 0$ define

$$
R_{s}(N, \gamma)=\sum_{B}\left(\prod_{\psi \in B} \delta_{\psi}\right)(\gamma)
$$

where the sum is over all asymmetric subsets $B$ of $N \cup \bar{N}$ with $|B|=s$. We adopt the convention that

$$
R_{0}(N, \gamma)= \begin{cases}1 & \text { if } \gamma=1, \\ 0 & \text { otherwise },\end{cases}
$$

and for $s>0$, if the sum is empty, we set $R_{s}(N, \gamma)=0$. Thus, if there is a constant $\beta>0$ such that $|g(\gamma)| \leq \beta$ for all $\gamma$ in $N$ then

$$
\left|C_{A}^{s}(\psi)\right| \leq R_{s}(A, \psi) \beta^{s}
$$

for all $\psi$ in $\widehat{K}(s=0,1, \ldots)$ and $A$ any symmetric subset of $N \cup \bar{N}$. The above inequality is immediate from the observation that $C_{A}^{s}(\psi)$ is formed by summing over asymmetric subsets $B$ of $A$ and the fact that $g$ is hermitian. We now have the following estimates:

$$
\begin{gathered}
\left|C_{A}(\gamma)-g(\gamma)\right| \leq \sum_{s=2}^{\infty} R_{s}(P, \gamma) \beta^{s} \text { for } \gamma \in P, \\
\left|C_{A}(1)-1\right| \leq \sum_{s=2}^{\infty} R_{s}(P, 1) \beta^{s}
\end{gathered}
$$

and

$$
\left|C_{A}(\gamma)\right| \leq \sum_{s=2}^{\infty} R_{s}(P, \gamma) \beta^{s} \text { for } \gamma \notin P \cup\{1\} .
$$

Certainly, if $g(\gamma)=\beta>0$ for all $\gamma$ in $P$ (and hence for all $\gamma$ in $Q=P \cup \bar{P}$ since $g$ is hermitian) we have

$$
P_{A}=\sum_{\gamma \in \widehat{K}} \gamma\left(\sum_{s=0}^{\infty} R_{s}(A, \gamma) \beta^{s}\right)
$$

\section{Sets of independence}

In this section we will restrict our attention to subsets of the dual of a compact abelian hypergroup. A subset $E$ of $\widehat{K}$ is said to be independent if 
$1 \notin E$ and if the following two conditions are satisfied for any finite subset $F=\left\{\gamma_{1}, \ldots, \gamma_{n}\right\}$ of $E$ :

(i) for each $\gamma \in E \backslash F,\langle F\rangle \cap\langle\gamma\rangle=\{1\}$;

(ii) $\left\{\psi_{1}\right\} *\left\{\psi_{2}\right\} * \cdots *\left\{\psi_{n}\right\}$ is a singleton in $\widehat{K}$ for all $\psi_{i} \in\left\langle\gamma_{i}\right\rangle \quad(i=$ $1, \ldots, n)$.

Of course (ii) is immediate if $\widehat{K}$ is a group but is quite restrictive if $\widehat{K}$ is only a hypergroup. If $K$ is a compact abelian group then the definition of independence given above corresponds to the usual definition of independence. Further examples may be found in any compact abelian hypergroup whose dual $\widehat{K}$ contains a direct product of subhypergroups. The next proposition provides two important properties of independent sets.

Proposition 3.1. Let $K$ be a compact abelian hypergroup with $E \subseteq \hat{K}$ independent. Let $F=\left\{\gamma_{1}, \ldots, \gamma_{k}\right\}$ be any finite subset of $E, H=\langle F\rangle$ and $\mu_{i} \in M(H)$ such that $\operatorname{supp}\left(\mu_{i}\right) \subseteq F_{i} \equiv\left\langle\gamma_{i}\right\rangle$ for $i=1,2, \ldots, k$. Then each element $\psi$ in $H$ can be written uniquely in the form $\psi=\left\{\psi_{1}\right\} *\left\{\psi_{2}\right\} * \cdots *$ $\left\{\psi_{n}\right\}$ where $\psi_{i} \in F_{i}(i=1,2, \ldots, k)$ and

$$
\mu(\psi)=\prod_{i=1}^{k} \mu_{i}\left(\psi_{i}\right)
$$

where $\mu=\mu_{1} * \cdots * \mu_{k}$.

Proof. Clearly, every element in $H$ can be written as a product of elements from each of the $F_{i}(i=1, \ldots, k)$. Suppose that

$$
\left\{\psi_{1}\right\} *\left\{\psi_{2}\right\} * \cdots *\left\{\psi_{k}\right\}=\psi=\left\{\phi_{1}\right\} *\left\{\phi_{2}\right\} * \cdots *\left\{\phi_{k}\right\}
$$

where $\psi_{i}, \phi_{i} \in F_{i}(i=1, \ldots, k)$. Then $\left\{\psi_{1}\right\} *\left\{\bar{\phi}_{1}\right\} \cap\left(\left\{\bar{\psi}_{2}\right\} *\left\{\phi_{2}\right\} * \cdots *\right.$ $\left.\left\{\bar{\psi}_{k}\right\} *\left\{\phi_{k}\right\}\right) \neq \phi$, so condition (i) of independence implies $1 \in\left\{\psi_{1}\right\} *\left\{\bar{\phi}_{1}\right\}$ and hence $\psi_{1}=\phi_{1}$. Uniqueness now follows by induction. Now, for any $\psi \in H$ and $\mu=\mu_{1} * \cdots * \mu_{k}$ with $\operatorname{supp}\left(\mu_{i}\right) \subseteq F_{i}$ we have

$$
\mu(\phi)=\sum_{\phi^{(1)} \in H} \cdots \sum_{\phi^{(k)} \in H}\left(\prod_{i=1}^{k} \delta_{\phi^{(i)}}\right)(\psi)\left(\prod_{j=1}^{k} \mu_{j}\left(\phi^{(j)}\right)\right) .
$$

Since $\mu_{j}$ is supported on $F_{j}$ we have $\mu_{j}\left(\phi^{(j)}\right)=0$ unless $\phi^{(j)} \in F_{j}$. Thus

$$
\mu(\psi)=\sum_{\sigma_{1} \in F_{1}} \cdots \sum_{\sigma_{k} \in F_{k}}\left(\prod_{i=1}^{k} \delta_{\sigma_{i}}\right)(\psi)\left(\sum_{j=1}^{k} \mu_{j}\left(\sigma_{j}\right)\right) .
$$


However, $\psi$ is written uniquely as a product of elements from the $F_{i}$ ( $i=$ $1, \ldots, k)$ so we conclude

$$
\mu(\psi)=\prod_{j=1}^{k} \mu_{j}\left(\sigma_{j}\right)
$$

It is interesting to compare the above proposition with $[2,4.2]$ where it is shown that for probability measures $\mu$ and $\nu$ on a hypergroup $K$ with $X, Y \subseteq K$ we have $\mu * \nu(X * Y) \geq \mu(X) \nu(Y)$.

A subset $E$ of $\widehat{K}$ is called quasi-independent if for each finite subset $A$ of $E, R_{s}(A, 1)=0$ for $s=1,2, \ldots$. This agrees with the definition of quasiindependence given by Pisier [17] for compact abelian groups and can easily be extended to agree with the definition given by Wilson [24, 5.3] for compact nonabelian groups. We call $E \subseteq \widehat{K}$ a Rider set if there is a constant $B>0$ such that $R_{s}(E, 1) \leq B^{s} \quad(s=0,1,2, \ldots)$ and a finite union of Rider sets is called a Stechkin set (compare with [11, 2.13]). For the properties we have described above, namely

(a) independent,

(b) quasi-independent,

(c) Rider,

(d) Stechkin,

it is immediate that $(a) \Rightarrow(b) \Rightarrow(c) \Rightarrow(d)$.

While infinite independent sets may not exist in the dual of an infinite compact abelian hypergroup (see Example 8.1) the next theorem shows that quasi-independent sets are found in abundance.

THEOREM 3.2. Let $K$ be a compact abelian hypergroup with $P$ an infinite subset of $\widehat{K}$. Then $P$ contains an infinite quasi-independent set.

PROoF. Select an arbitrary element $\gamma_{1} \in P \backslash\{1\}$. Having selected $\gamma_{1}, \ldots$, $\gamma_{k}$ select $\gamma_{k+1} P \backslash F$ where $F$ is the finite set of all elements of $\widehat{K}$ which belong to any of the sets $\left\{\psi_{1}\right\} *\left\{\psi_{2}\right\} * \cdots *\left\{\psi_{k}\right\}$ where $\psi_{i} \in\left\{1, \gamma_{i}, \bar{\gamma}_{i}\right\} \quad(i=$ $1,2, \ldots, k)$. This construction leads to an infinite set $E=\left\{\gamma_{1}, \gamma_{2}, \ldots\right\} \subseteq$ $P$. Let $A$ be a finite subset of $E$, say $A=\left\{\gamma_{n_{1}}, \gamma_{n_{2}}, \ldots, \gamma_{n_{s}}\right\}$, where $n_{1}<n_{2}<\cdots<n_{s}$. If $R_{s}(A, 1) \neq 0$ then $1 \in\left\{\psi_{n_{1}}\right\} *\left\{\psi_{n_{2}}\right\} * \cdots *\left\{\psi_{n_{s}}\right\}$ where $\psi_{n_{i}} \in\left\{\gamma_{n_{i}}, \bar{\gamma}_{n_{i}}\right\}$. But this implies either $\gamma_{n_{s}} \in\left\{\psi_{n_{1}}\right\} *\left\{\psi_{n_{2}}\right\} * \cdots *\left\{\psi_{n_{s-1}}\right\}$ or $\gamma_{n_{s}} \in\left\{\bar{\psi}_{n_{1}}\right\} *\left\{\bar{\psi}_{n_{2}}\right\} * \cdots *\left\{\bar{\psi}_{n_{s-1}}\right\}$, contrary to the construction of $E$. Thus $E$ is an infinite quasi-independent subset of $P$. 


\section{Some sufficient conditions for Sidonicity}

We call a subset $E$ of $\widehat{K}$ bounded if the set $\left\{k_{\gamma}: \gamma \in E\right\}$ is bounded where $k_{\gamma}^{-1}=\int_{K} \gamma \bar{\gamma} d m$. In general, the dual of a compact abelian hypergroup need not be bounded. Indeed, the dual object for a compact non-abelian group may not have any infinite bounded subsets (for example, see [8]). For bounded subsets of $K$ we are able to extend the group result that Stechkin sets are Sidon sets. First, we need a few preliminary results. The first lemma generalizes a lemma due to Rider [18].

LEMMA 4.1. If $E$ is a bounded Rider set with bound $C$ and $1 \notin E$ there is a constant $B>0$ such that for $s \geq 0$

$$
R_{s}(E, \gamma) \leq B^{s} \text { for all } \gamma \text { in } E
$$

and

$$
k_{\gamma}^{-1} R_{s}(E, \gamma) \leq\left(B C^{-1}\right)^{s}
$$

Proof. Let $B_{1}$ be the Rider constant for $E$. Let $\beta=\left(2 B_{1}\right)^{-1}$ and define $g(\gamma)=\beta$ for $\gamma$ in $E$. For $A$ an arbitrary finite subset of $E$ define the Riesz product

$$
P_{A}=\sum_{\gamma \in \widehat{K}} \gamma\left(\sum_{s=0}^{\infty} \beta^{s} R_{s}(A, \gamma)\right) .
$$

Since $P_{A}=\prod r_{\gamma},|\gamma(t)| \leq 1$ for all $t$ in $K$ and $\beta<1 / 2$, we see that $P_{A}$ is nonnegative. Thus for each $\gamma$ in $\widehat{K}$

$$
\left|\widehat{P}_{A}(\gamma)\right| \leq\left\|\widehat{P}_{A}\right\|_{u} \leq\left\|P_{A}\right\|_{1}=\widehat{P}_{A}(1) \leq \sum_{s=0}^{\infty} R_{s}(E, 1) B^{s} \leq 2 .
$$

Therefore, for $\gamma$ in $E$,

$$
\sum_{s=0}^{\infty} R_{s}(E, \gamma) \beta^{s} \leq 2 k_{\gamma} \leq 2 C,
$$

so $R_{s}(E, \gamma) \leq\left(4 C B_{1}\right)^{s}=B^{s}$ for $B=4 C B_{1}$. If $\gamma$ is not in $E$ then

$$
\sum_{s=0}^{\infty} k_{\gamma}^{-1} R_{s}(E, \gamma) \beta^{s} \leq 2
$$

and hence $k_{\gamma}^{-1} R_{s}(E, \gamma) \leq\left(4 B_{1}\right)^{s}=\left(B C^{-1}\right)^{s}$. 
LEMMA 4.2. Let $E$ be a bounded symmetric Rider set with 1 not in $E$ and let $B \geq 1$ be such that $R_{s}(E, \gamma) \leq B^{s}$ for all $\gamma$ in $E$ and $s \geq 0$. If $0<\varepsilon<1, g$ is hermitian on $E$ and $\|g\|_{\infty} \leq 1$, then there exists $\mu$ in $M^{+}(K)$ such that

$$
\begin{gathered}
\|\mu\| \leq \varepsilon+2 B^{2} C^{2} / \varepsilon \\
|\widehat{\mu}(\gamma)-g(\gamma)| \leq \varepsilon \text { for } \gamma \text { in } E
\end{gathered}
$$

and

$$
|\widehat{\mu}(\gamma)| \leq \varepsilon \text { for } \gamma \text { not in } E \cup\{1\},
$$

where $C$ is a bound for $\left\{k_{\gamma}: \gamma \in E\right\}$.

Proof. Let $\beta=\varepsilon\left(2 B^{2} C\right)^{-1}$ and define $g_{1}(\gamma)=\left(k_{\gamma} C^{-1}\right) \beta g(\gamma)$ for $\gamma$ in $E$ and note that $\left|g_{1}(\gamma)\right| \leq \beta \leq 1 / 2$. Consider the Riesz product $P_{A}$ for $A$ an arbitrary finite subset of $E$ with function $g_{1}$. If $\gamma$ is in $E$ we have

$$
\begin{aligned}
\sum_{s=2}^{\infty} R_{s}(E, \gamma) \beta^{s} & \leq \sum_{s=2}^{\infty}(B \beta)^{s}=\sum_{s=2}^{\infty}\left[\varepsilon(2 B C)^{-1}\right]^{s} \\
& \leq 2\left[\varepsilon(2 B C)^{-1}\right]^{2}=\varepsilon \beta C^{-1} .
\end{aligned}
$$

If $\gamma$ is in $\widehat{K}-E$ then from Lemma 4.1 we have $k_{\gamma}^{-1} R_{s}(E, \gamma) \leq\left(B C^{-1}\right)^{s}$ and so

$$
\sum_{s=0}^{\infty} k_{\gamma}^{-1} R_{s}(E, \gamma) \beta^{s} \leq \sum_{s=2}^{\infty}\left(B C^{-1} \beta\right)^{s} \leq \varepsilon \beta C^{-3} \leq \varepsilon \beta C^{-1} .
$$

Now applying the above inequality along with estimates (2.1) and (2.2) we have

$$
\left\|P_{A}\right\|_{1}=\widehat{P}_{A}(1) \leq 1+\sum_{s=2}^{\infty} R_{s}(E, 1) \beta^{s} \leq 1+\varepsilon \beta C^{-1}
$$

and

$$
\left|C_{A}(\gamma)-g_{1}(\gamma)\right| \leq \varepsilon \beta C^{-1} \text { for } \gamma \text { in } E .
$$

Now $\widehat{P}_{A}(\gamma)=k_{\gamma}^{-1} C_{A}(\gamma)$ so for $\gamma$ in $A$

$$
\left|k_{\gamma} \widehat{P}_{A}(\gamma)-k_{\gamma} \beta C^{-1} g(\gamma)\right| \leq \varepsilon \beta C^{-1}
$$

or

$$
\left|\widehat{P}_{A}(\gamma)-\beta C^{-1} g(\gamma)\right| \leq \varepsilon \beta C^{-1} k_{\gamma}^{-1} \leq \varepsilon \beta C^{-1}
$$

and using (2.3) for $\gamma$ not in $A \cup\{1\}$, we obtain

$$
\left|k_{\gamma} \widehat{P}_{A}(\gamma)\right| \leq \sum_{s=2}^{\infty} R_{s}(E, \gamma) \beta^{s} \text { or }\left|\widehat{P}_{A}(\gamma)\right| \leq \varepsilon \beta C^{-1} .
$$


By Alaoglu's theorem, the net $\left\{P_{A}\right\}$ in $M^{+}(K)$ has a weak-* cluster point $\nu$ in $M^{+}(K)$ such that

$$
\begin{gathered}
\|\nu\| \leq 1+\varepsilon \beta C^{-1}, \\
|\widehat{\nu}(\gamma)-\beta g(\gamma)| \leq \varepsilon \beta C^{-1} \text { for } \gamma \text { in } E, \\
|\widehat{\nu}(\gamma)| \leq \varepsilon \beta C^{-1} \text { for } \gamma \text { not in } E \cup\{1\} .
\end{gathered}
$$

Finally, let $\mu=C \beta^{-1} \nu$.

THEOREM 4.3. Let $E$ be a bounded symmetric Stechkin set with 1 not in $E$. Then there is a constant $\alpha$ with the following property. If $0<\varepsilon<1, g$ is hermitian on $E$, and if $\|g\|_{\infty} \leq 1$, then there exists $\mu$ in $M^{+}(K)$ such that

$$
\begin{gathered}
\|\mu\| \leq \varepsilon+\alpha \varepsilon^{-1}, \\
|\widehat{\mu}(\gamma)-g(\gamma)| \leq \varepsilon \text { for all } \gamma \text { in } E,
\end{gathered}
$$

and

$$
|\widehat{\mu}(\gamma)| \leq \varepsilon \text { for } \gamma \text { not in } E \cup\{1\} \text {. }
$$

Proof. Use Lemma 4.2 and imitate the argument in [11, 2.18].

We are now able to establish the following corollaries.

COROLlARY 4.4. Every bounded Stechkin set in a compact abelian hypergroup is a Sidon set.

Proof. Apply Theorem 4.3 and $[22,2.5]$.

COROLlARY 4.5. Every bounded infinite subset of $\widehat{K}$ contains an infinite Sidon set which is also a $\Lambda$-set.

PROof. This follows easily from Theorem 3.2, Corollary 4.4 and [22, 3.5]. In order to remove the hypothesis of boundedness used above we will require a greater degree of independence for $E$. The next theorem is a hypergroup version of Kronecker's theorem for locally compact abelian groups (compare with [21, 5.13]).

THEOREM 4.6. Suppose $K$ is a compact abelian strong hypergroup and let $F=\left\{\gamma_{1}, \ldots, \gamma_{k}\right\}$ be a finite subset of an independent set $E$ in $\widehat{K}$. If $\left\langle\gamma_{i}\right\rangle=F_{i}$ and $\phi_{i} \in F_{i}$ then there exists $x$ in $K$ such that

$$
\gamma_{i}(x)=\phi_{i}\left(\gamma_{i}\right) \quad \text { for } i=1,2, \ldots, k .
$$


Proof. Since $E$ is independent, Proposition 3.1 shows that each $\psi$ in $\langle F\rangle$ can be written uniquely in the form $\left\{\psi_{1}\right\} *\left\{\psi_{2}\right\} * \cdots *\left\{\psi_{k}\right\}$. Define a function $h$ on $\langle F\rangle$ by

$$
h\left(\psi_{1} \cdots \psi_{k}\right)=\prod_{i=1}^{k} \phi_{i}\left(\psi_{i}\right) .
$$

Given $\psi=\prod_{i=1}^{k} \psi_{i}$ and $\sigma=\prod_{i=1}^{k} \sigma_{i}$ in $\langle F\rangle$ we have

$$
\begin{aligned}
h(\psi \sigma) & =\sum_{\chi \in\langle F\rangle} h(\chi) \delta_{\psi} * \delta_{\sigma}(\chi) \\
& =\sum_{\chi \in\langle F\rangle}\left(\prod_{i=1}^{k} \phi_{i}\left(\chi_{i}\right)\right)\left(\prod_{j=1}^{k} \delta_{\psi_{j}} * \prod_{j=1}^{k} \delta_{\sigma_{j}}\right)(\chi) \\
& =\sum_{\chi \in\langle F\rangle}\left(\prod_{i=1}^{k} \phi_{i}\left(\chi_{i}\right)\right)\left(\prod_{j=1}^{k}\left(\delta_{\psi_{j}} * \delta_{\sigma_{j}}\right)\right)(\chi) .
\end{aligned}
$$

Applying Proposition 2.1 we have

$$
\begin{aligned}
h\left(\psi_{\sigma}\right) & =\sum_{x \in\langle F\rangle}\left(\prod_{i=1}^{k} \phi_{i}\left(\chi_{i}\right)\left(\delta_{\psi_{i}} * \delta_{\sigma_{i}}\right)\left(\chi_{i}\right)\right) \\
& =\sum_{\chi_{1} \in F_{1}} \cdots \sum_{\chi_{k} \in F_{k}}\left(\prod_{i=1}^{k} \phi_{i}\left(\chi_{i}\right) \delta_{\psi_{i}} * \delta_{\sigma_{i}}\left(\chi_{i}\right)\right) \\
& =\prod_{i=1}^{k}\left(\sum_{\chi_{i} \in F_{i}} \phi_{i}\left(\chi_{i}\right) \delta_{\psi_{i}} * \delta_{\sigma_{i}}\left(\chi_{i}\right)\right)=\prod_{i=1}^{k} \phi_{i}\left(\psi_{i}\right) \phi_{i}\left(\sigma_{i}\right) \\
& =h(\psi) h(\sigma) .
\end{aligned}
$$

Hence $h$ is a character on $\widehat{K}$. Since $K$ is a strong hypergroup $(\widehat{\widehat{K}}) \simeq K$ so the points of $K$ separate $\widehat{K}$ thus they also separate the elements of $\langle F\rangle$. Thus there is an $x$ in $K$ such that $\psi(x)=h(\psi)$ for all $\psi$ in $\langle F\rangle$. In particular, we have

$$
\gamma_{i}(x)=h\left(\gamma_{i}\right)=\phi_{i}\left(\gamma_{i}\right)
$$

We are now in a position to show that independent subsets of $K$ are Sidon sets under the assumption that $K$ is a strong hypergroup. The proof is similar to the proof of $[14,4.1]$.

THEOREM 4.7. If $K$ is a compact abelian strong hypergroup with $E$ an independent subset of $\widehat{K}$ then $E$ is a Sidon set. 
Proof. Let $f \in \operatorname{Trig}_{E}(K)$ where $f=\sum_{\gamma \in F} k_{\gamma} a_{\gamma} \gamma$ and $F$ is a finite subset of $E$. Select a complex number $\theta$ such that $\theta^{4}=1$ and a subset $A$ of $F$ such that

$$
A=\left\{\gamma \in F: \operatorname{Re}\left(\theta a_{\gamma}\right) \geq 0\right\}
$$

and

$$
\operatorname{Re}\left(\sum_{\gamma \in A} k_{\gamma} a_{\gamma} \theta\right) \geq \frac{1}{4} \sum_{\gamma \in F} k_{\gamma}\left|a_{\gamma}\right|=\frac{1}{4}\|\hat{f}\|_{1} .
$$

Let $P=\left\{\gamma \in F \backslash A: \operatorname{Im}\left(\theta a_{\gamma}\right) \geq 0\right\}$ and $N=\left\{\gamma \in F \backslash A: \operatorname{Im}\left(\theta a_{\gamma}\right)<0\right\}$. For each $\gamma$ in $A$ select $\phi_{\gamma}$ to be the trivial character in $\langle\gamma\rangle$. If $\gamma$ is in $P$ then the orthogonality condition $\int_{K} \gamma d m=0$ implies $\operatorname{Re}(\gamma(x))<0$ for some $x$ in $K$. If $\operatorname{Im}(\gamma(x)) \geq 0$ let $y=\check{x}$, otherwise let $y=x$. Select $\phi_{y}=\hat{y}$ in $\langle\gamma\rangle^{\sim}$. Similarly, for $\gamma$ in $N$ select $y$ so that $\operatorname{Re}(\gamma(y))<0$ and $\operatorname{Im}(\gamma(y)) \geq 0$ and set $\phi_{\gamma}=\hat{y}$ in $\langle\gamma\rangle$. Now we apply Theorem 4.6 to find an $x$ in $K$ such that $\gamma(x)=\phi_{\gamma}(\gamma)$ for all $\gamma$ in $F$. Thus

$$
\begin{aligned}
\|f\|_{u} & \geq|f(x)| \geq \operatorname{Re}(\theta f(x))=\operatorname{Re}\left(\sum_{\gamma \in F} k_{\gamma} \theta a_{\gamma} \gamma(x)\right) \\
& \geq \operatorname{Re}\left(\sum_{\gamma \in A} k_{\gamma} \theta a_{\gamma}\right) \geq \frac{1}{4}\|\hat{f}\|_{1} .
\end{aligned}
$$

Thus $E$ is a Sidon set with Sidon constant at most 4 , by [22, 2.2].

\section{A necessary condition for Sidonicity}

The previous section provided two distinct sets of sufficient conditions to ensure a subset of $\widehat{K}$ is a Sidon set. In this short section we provide a necessary condition which must be satisfied by any infinite Sidon set. Note that in this section we do not require that $\widehat{K}$ is a hypergroup.

THEOREM 5.1. Let $K$ be a compact abelian hypergroup and $E$ an infinite subset of $\widehat{K}$. If $E$ is a Sidon set then $\left\{x \in K: \hat{\delta}_{x} \notin c_{0}(E)\right\}$ is not a finite subhypergroup of $K$.

Proof. Suppose $H=\left\{x \in K: \widehat{\delta}_{x} \notin c_{0}(E)\right\}$ is a finite subhypergroup. This will lead to a contradiction. Each $\gamma \in E$, when restricted to $H$, is in the finite set $\hat{H}$ so there must be an infinite number of $\gamma$ in $E$ which agree on $H$. Let $P$ denote this infinite subset of $E$. We will in fact show that $P$ 
is not a Sidon set, contradicting the assumption that $E$ is a Sidon set. Let $\mu \in M(K)$ and consider $\widehat{\mu}(\gamma)$ for each $\gamma$ in $P$. Then

$$
\widehat{\mu}(\gamma)=\int_{H} \bar{\gamma} d \mu+\int_{K \backslash H} \bar{\gamma} d \mu .
$$

However, for $t \in K \backslash H$, we have $\widehat{\delta}_{t} \in c_{0}(P)$ and each $\gamma$ in $P$ restricts to the same element of $\widehat{H}$ so there is a constant $B_{\mu}$ such that

$$
\widehat{\mu}(\gamma)=B_{\mu}+\int_{K \backslash H} \bar{\gamma} d \mu \text {. }
$$

Therefore, there is no measure $\mu$ in $M(K)$ with $\widehat{\mu}$ taking arbitrary values \pm 1 on $P$ so $P$ is not a Sidon set by $[22,2.5]$.

The above proof is a generalization of a proof given by Rider [19, Theorem 9]. The above theorem is also similar to a necessary condition for Helson sets on $K$ (see [23, 4.3]). Any compact abelian hypergroup which is $n$ fold absolutely continuous (see [20] for more details on such hypergroups) and has finite center does not satisfy the necessary condition provided in the previous theorem. Hence, Theorem 5.1 generalizes $[23,3.2]$ without the additional assumption that $\widehat{K}$ is a hypergroup.

\section{Compact lacunarity for groups}

In this section we make explicit a result which appears implicitly in the important paper of Pisier [16]. In an earlier paper [15], Pisier showed the equivalence of Sidon sets and $\Lambda$ sets for compact abelian groups. In the hope of using commutative techniques so study the compact nonabelian case, Parker [14] introduced the notion of central Sidon sets and this was followed by a discussion of central $\Lambda$ sets by Benke [1]. The appropriate definitions for central lacunary sets are found by replacing the function spaces given in the definition of Sidon or $\Lambda$ sets by their respective centers. Rider [19] has shown there are central Sidon sets which are not central $\Lambda$ sets.

Pisier [16] establishes the surprising result that Sidonicity for a compact group can be determined strictly in terms of central functions. Specifically, he establishes the equivalence of the following two statements for $P \subseteq \widehat{G}$, where $G$ is a compact group:

(i) $P$ is a Sidon set;

(ii) there exists a constant $C$ such that, for all $\left(a_{\chi}\right)_{\chi \in P} \in l^{2}(P)$,

$$
\left\|\sum_{\chi \in P} a_{\chi} \chi\right\|_{\psi_{2}} \leq C\left(\sum_{\chi \in P}\left|a_{\chi}\right|^{2}\right)^{1 / 2}
$$


where $\psi_{2}(t)=e^{t^{2}}-1$ and $\|f\|_{\psi_{2}}=\inf \left\{c>0: \int_{G} \psi(f / c) d m \leq 1\right\}$.

The above result easily implies the equivalence of Sidon sets and central $\Lambda$ sets for compact groups, as the following theorem establishes.

THEOREM 5.1. Let $G$ be a compact group and $P \subseteq \widehat{G}$. The following are equivalent:

(i) $P$ is a Sidon set;

(ii) $P$ is a $\Lambda$ set;

(iii) $P$ is a central $\Lambda$ set.

Proof. If $P$ is a Sidon set then $P$ is a $\Lambda$ set $[7,37.25]$, and so (i) implies (ii). Clearly (ii) implies (iii). Next, we assume $P$ is central $\Lambda$. Suppose $f$ has Fourier series $\sum_{\chi \in P} a_{\chi} \chi$ where $\sum_{\chi \in P}\left(a_{\chi}\right)^{2}<\infty$. Then $f$ is a central function on $G$. By $[12,5.2 .11]$ we have

$$
N_{2}(f) \leq K \sup _{n>1} 1 / \sqrt{n}\|f\|_{n}
$$

where $K$ is a constant and $N_{2}(f)=\inf \left\{c>0: \int e^{(f / c)^{2}} d m \leq 2\right\}$. Thus $\|f\|_{\psi_{2}} \leq K \sup _{n \geq 1} 1 / \sqrt{n}\|f\|_{n}$. But $P$ is central $\Lambda$ so

$$
\|f\|_{p} \leq L \sqrt{p}\|f\|_{2}
$$

for some constant $L$ and all $p>2$. Thus

$$
K p^{-1 / 2}\|f\|_{p} \leq K L\|f\|_{2}
$$

for all $p>2$. Since $G$ is compact, we have $\|f\|_{p} \leq\|f\|_{2}$ for all $1 \leq p \leq 2$, so

$$
K \sup _{p \geq 1} 1 / \sqrt{p}\|f\|_{p} \leq K L\|f\|_{2}
$$

and hence $\|f\|_{\psi_{2}} \leq K L\|f\|_{2}$. This implies $P$ is a Sidon set by Pisier's result.

As a consequence of the above, lacunarity on compact groups can be studied by examining central lacunarity on compact groups. In Section 7 we will place these remarks in the context of compact abelian hypergroups and use results from earlier sections to provide information about lacunarity on compact groups.

\section{Applications to lacunarity on compact groups}

Since central Sidon (central $\Lambda$, central $\Lambda_{p}$ ) on a compact (not necessarily abelian) group $G$ is equivalent to Sidon $\left(\Lambda, \Lambda_{p}\right)$ on the corresponding compact abelian hypergroup of conjugacy classes of $G$ (see [22, 2.93]), written 
$G_{I}$, we may apply hypergroup results to provide information about central lacunarity on compact groups. Furthermore, Theorem 6.1 shows that $\widehat{G}_{I}$ retains enough structure of $\widehat{G}$ to decide some lacunary questions about $G$. We have the advantage of using abelian techniques when studying $G_{I}$ but of course there is the added complexity of the more general hypergroup structure. We will illustrate some of these ideas in this section.

REMarK 7.1. We begin by discussing some questions posed by Parker [14]. Parker generalized the notion of independence on compact abelian hypergroups with the notion of $I$-sets on the dual of a compact group. He asked whether a better definition then $I$-set could be devised. The definition of independence given in Section 3 completely agrees with the definition of independence on compact abelian groups, and the proof of Theorem 4.7 shows that every independent set is an $I$-set in the sense of Parker. Theorem 4.7 also provides a generalization of $[14,4.2]$, which says that any infinite direct product of compact groups admits an infinite central Sidon set.

REMARK 7.2. Parker also asked whether any additional hypothesis on a central Sidon set would be enough to ensure it is a Sidon set. It has apparently been known for some time that boundedness and central Sidon is enough to imply Sidon but as far as the author knows this has never appeared in the literature. We may use Theorem 6.1 and $[22,3.5]$ to see that boundedness is sufficient for central Sidon to imply Sidon. He also asked whether an infinite bounded subset of the dual object must contain an infinite Sidon set. An affirmative answer is provided by an application of Corollary 4.5 and Theorem 6.1 or follows from results in Hutchinson [8].

REMARK 7.3. Parker also asked whether there are any nonabelian infinite compact groups such that every central Sidon set is a Sidon set. An affirmative answer was provided to this question by Rider [19, Theorem 9] when he showed that compact connected semi-simple Lie groups admit only finite central Sidon sets. The next theorem provides a complete characterization of compact connected groups with this property.

THEOREM 7.4. Let $G$ be a compact connected group. Every central Sidon set for $G$ is a Sidon set if and only if $G$ is a Lie group.

Proof. Without loss of generality, we may assume $G$ is 2 nd countable and write $G=G_{0} / N$ where

$$
G_{0}=\prod_{n \in I} S_{n} \times A
$$

and where the $S_{n}$ are compact connected simple Lie groups, $A$ is a compact connected abelian group and $N$ is a closed normal subgroup of $G_{0}$ with 
$N \cap A=N \cap S_{n}=\{e\}$ (see, for example, [19]). First, we assume $G$ is a Lie group, that is, that $I$ is finite. Let $E \subseteq \widehat{G}$ be a central Sidon set. From Remark 7.2, it suffices to show $E$ is bounded. Let $S=\prod_{n \in I} S_{n}$ so that $G_{0} / A \simeq S$ and $\left(G_{0} / A\right)^{\wedge} \simeq S^{\wedge}$ (as hypergroups). The characters in $\hat{A}$ are group characters, that is, 1-dimensional, so on each of the cosets $\sigma \widehat{A}$, $\sigma \in \widehat{G}_{0}$ the representations have a fixed degree. If $E$ is not bounded then the members of $E$ would lie in an infinite number of distinct cosets of $\widehat{S}$ and would then in turn form an infinite central Sidon set for $\widehat{S}$, contrary to Rider's result [19, Theorem 9] that compact connected semi-simple Lie groups admit no infinite central Sidon sets. Thus $E$ is bounded, which implies $E$ is also a Sidon set.

Next, we assume $G$ is not a Lie group, in which case $I$ is infinite. Then there is a closed normal subgroup $H$ of $G$ such that $G / H=\prod_{n \in I}\left(S_{n} / Z_{n}\right)$, where $Z_{N}$ is the center of $S_{n}$. Each of the groups $S_{n} / Z_{n}$ is a compact connected simple Lie group so a result of Cecchini [4] implies that for each $n=1,2, \ldots,\left(S_{n} / Z_{n}\right)^{\sim}$ contains no infinite local central $\Lambda_{4}$ sets. In particular, in $\left(S_{n} / Z_{n}\right)^{n}$ we may select $\sigma_{n}$ so that $\left\|\chi_{\sigma_{n}}\right\|_{4}>n\left\|\chi_{\sigma_{n}}\right\|_{2}$. If $\pi_{n}$ denotes the natural projection of $G / H$ then the set $E \equiv\left\{\chi_{n}: n \in I\right\}$ where $\chi_{n}=\pi_{n} \chi_{\sigma_{n}}$ is a central Sidon set by either $[14,6.11]$ or Theorem 4.7. However, the construction of $E$ implies $E$ is not a local central $\Lambda_{4}$ set so that $E$ is not a Sidon set. Thus $E$ is a central Sidon set which is not a Sidon set.

The setting of compact connected groups seems to be particularly well suited to analysis in terms of the associated conjugacy class hypergroup as evidenced by recent work of McMullen [13].

As already noted in Section 6, Pisier has shown that Sidon and $\Lambda$ sets are equivalent for compact abelian groups. However, central Sidon does not (in general) imply central $\Lambda$. We may rephrase the previous theorem to characterize among compact connected groups $G$, all the compact connected conjugacy class hypergroups $G_{I}$ for which Sidon and $\Lambda$ are equivalent.

COROLlary 7.5. Let $G$ be a compact connected group and $G_{I}$ its conjugacy class hypergroup. The following are equivalent:

(i) Sidon sets and $\Lambda$ sets are equivalent for $G_{I}$;

(ii) $G$ is a Lie group;

(iii) $G_{I}$ is finitely generated.

REMARK 7.6. Hutchinson $[8,2.4]$ has shown that if $G$ is a compact group with $E \subseteq G$ a Rider set, and $d_{\sigma}=n$ (fixed) for all $\sigma$ in $E$, then $E$ is a Sidon set. In fact, $E$ is a uniformly approximable Sidon set, from a result 
of Dunkl and Ramirez [5]. Combining Theorem 4.3 and Theorem 6.1, we have a complete generalization of Hutchinson's result. Hutchinson called a compact group $G$ tall if no infinite subsets of $\widehat{G}$ are bounded. He then goes on to show that every compact group which is not tall admits an infinite Sidon set. This result also follows easily from Corollary 4.5 and Theorem 6.1. It should be noted that Cartwright and McMullen [3] have characterized those compact connected groups which admit infinite Sidon sets.

REMARK 7.7. An important open question in the area of lacunarity on compact abelian groups is whether every Sidon set can be written as a finite union of quasi-independent sets (see [17]). The situation for compact groups is considerably more complicated. For example, not every quasi-independent set in a compact group is necessarily central Sidon, let alone Sidon. This can be seen easily by considering an infinite connected semi-simple Lie group $G$. Then $\widehat{G}_{I}$ and hence $\widehat{G}$ contain an infinite number of quasi-independent sets, by Theorem 3.2, but $G$ admits no infinite central Sidon sets as already discussed. Wilson $[24,5.8]$ has shown that every Sidon set for a compact connected group can be written as the union of a bounded Sidon set and a finite union of quasi-independent sets.

REMARK 7.8. Theorem 6.1 can be used to show that the union of two Sidon sets for a compact group is again a Sidon set (since the union of two central $\Lambda$ sets is a central $\Lambda$ set). This fact has also been established by Wilson [24, 4.3] for compact connected groups and is easily seen to follow from Pisier's characterization of Sidon sets for compact groups (see Section 6). Rider has shown [19, Example 8] that the union of two central Sidon sets need not be central Sidon.

ReMARK 7.9. Hewitt and Ross $[7,36.1]$ consider the compact group $G=$ $\prod_{i \in I} U\left(H_{i}\right)$, where $U\left(H_{i}\right)$ is the set of unitary operators on the Hilbert space $H_{i}$. For each $i$, let $\pi_{i}$ denote the projection of $G$ onto $U\left(H_{i}\right)$. Hewitt and Ross shown in $[7,36.2]$ that $P$ is a $\Lambda$ set. They go on to prove directly in $[7,37.5]$ that $P$ is also a Sidon set. By Theorem 6.1 , this result follows immediately from the fact that $P$ is a $\Lambda$ set.

\section{Examples}

EXAMPLE 8.1. We first consider the family of countable compact hypergroups introduced by Dunkl and Ramirez [6]. Let $K=\{0,1,2, \ldots, \infty\}$ be the 1 point compactification of the nonnegative integers and $\widehat{K}=\left\{\chi_{l}\right.$ : $l=0,1,2, \ldots\}$. The characters $\chi_{l}$ are real functions and $\chi_{n} \chi_{m}=\chi_{m}$ for $n<m[6,3.7]$. It follows easily that $\widehat{K} \backslash\left\{\chi_{0}\right\}$ is a quasi-independent 
set which is not independent. In fact, $\widehat{K}$ contains no infinite independent sets. Dunkl and Ramirez [5, 10.5] show that $K$ admits no infinite Helson or Sidon sets. This also follows easily from [23, 4.3] and Theorem 5.1 by observing $\widehat{\delta}_{x} \in c_{0}(\widehat{K})$ for all $x$ in $K \backslash\{\infty\}$. Note that $k_{\chi_{n}}=(1-a) a^{-n}$, where $0<a \leq 1 / 2$, so $\widehat{K}$ has no bounded infinite subsets.

EXAMPLE 8.2. This example involves the interesting Jacobi polynomial hypergroups introduced by Lasser [10]. Given real parameters $\alpha, \beta$ satisfying $\alpha \geq \beta>-1, \alpha+\beta+1 \geq 0$ and either $\beta \geq-1 / 2$ or $\alpha+\beta \geq 0$, Lasser constructs strong hypergroups $K(\alpha, \beta)=[-1,1]$ with $K(\alpha, \beta)^{\wedge}=$ $\{0,1,2, \ldots\}$ such that the characters in $K(\alpha, \beta)^{\wedge}$ are the Jacobi polynomials associated with $\alpha$ and $\beta$. In particular, $K(-1 / 2,-1 / 2)^{-}$consists of the Tchebichef polynomials of the first kind, $K(0,0)^{\wedge}$ gives the Legendre polynomials and $K(1 / 2,1 / 2)^{\wedge}$ the Tchebichef polynomials of the second kind. For the Tchebichef polynomials of the first kind we have $k_{n}=2$ for all $n \in K(-1 / 2,-1 / 2)^{\wedge}$, so every subset of the dual hypergroup is bounded. We may apply Corollary 4.5 to conclude that every infinite subset of $K(-1 / 2,-1 / 2)^{-}$contains an infinite Sidon set which is also a $\Lambda$-set. However, for the Legendre polynomials and the Tchebichef polynomials of the second kind we have $k_{n}=2 n+1$ and $k_{n}=(n+1)^{2}$ respectively. In these cases, no infinite subset of the dual is bounded. Moreover, if $x \in(-1,1)$ then $\lim _{n \rightarrow \infty} P_{n}(x)=0$ where $\left\{P_{n}\right\}_{n=1}^{\infty}$ is either the collection of Legendre polynomials or the Tchebichef polynomials of the second kind. Restating this in terms of the hypergroup structure, we have $\widehat{\delta}_{x} \in c_{0}(\widehat{K})$ for all $x \in K \backslash Z K$ where $K$ is either $K(0,0)$ or $K(1 / 2,1 / 2)$. Therefore, we may conclude from Theorem 5.1 that neither $K(0,0)$ nor $K(1 / 2,1 / 2)$ admit an infinite Sidon set. Indeed, neither do they admit any infinite Helson sets, by [23, 4.3].

\section{References}

[1] G. Benke, 'On the hypergroup structure of central $\Lambda(p)$-sets', Pacific J. Math. 50 (1974), 19-27.

[2] W. R.Bloom, 'Infinitely divisible measures on hypergroups', Probability measures on groups, Proc. Conf. Oberwolfach Math. Res. Inst. (Oberwolfach, 1981), pp. 1-15 (Lecture Notes in Mathematics 928, Springer, Berlin, Heidelberg, New York, 1982).

[3] D. I. Cartwright and J. R. McMullen, 'A structural criterion for the existence of infinite Sidon sets', Pacific J. Math. 97 (1981), 301-318.

[4] C. Cecchini, 'Lacunary Fourier series on compact Lie groups', J. Funct. Anal. 11 (1972), 191-203.

[5] C. F. Dunkl and D. E. Ramirez, 'Central Sidon sets of bounded representation type', Notices Amer. Math. Soc. 18A (1971), 1101. 
[6] C. F. Dunkl and D. E. Ramirez, 'A family of countable compact $P^{*}$-hypergroups', Trans. Amer. Math. Soc. 202 (1975), 339-356.

[7] E. Hewitt and K. A. Ross, Abstract harmonic analysis II, (Springer-Verlag, New York, 1970).

[8] M. F. Hutchinson, 'Non-tall compact groups admit infinite Sidon sets', J. Austral. Math. Soc. 23 (1977), 467-475.

[9] R. I. Jewett, 'Spaces with an abstract convolution of measures', Adv. in Math. 18 (1975), 1-101.

[10] R. Lasser, 'Orthogonal polynomials and hypergroups', Rend. Mat. (7) 3 (1983), 185-209.

[11] J. M. Lopez and K. A. Ross, Sidon sets, (Marcel Dekker, New York, 1975).

[12] M. B. Marcus and G. Pisier, Random Fourier series with applications to harmonic analysis, (Ann. of Math Studies, 101, Princeton Univ. Press, 1981).

[13] J. R. McMullen, 'On the dual object of a compact connected group', Math. Z. 185 (1984), 539-552.

[14] W. A. Parker, 'Central Sidon sets and central $\Lambda(p)$-sets', J. Austral. Math. Soc. 24 (1972), 62-74.

[15] G. Pisier, 'Lacunarité et processus gaussiens', C.R. Acad. Sci. Paris Ser. A 286 (1978), 1003-1006.

[16] G. Pisier, 'De nouvelles caractérisations des ensembles de Sidon', Mathematical Analysis and Applications, Part B, pp. 685-726 (Advances in Mathematics Supplementary Studies, 7B, 1981).

[17] G. Pisier, 'Arithmetic characterizations of Sidon sets', Bull. Amer. Math. Soc. 8 (1983), 87-89.

[18] D. Rider, 'Gap series on groups and spheres', Canad. J. Math. 18 (1966), 389-398.

[19] D. Rider, 'Central lacunary sets', Monatsh. Math. 76 (1972), 328-338.

[20] K. A. Ross, 'Centers of hypergroups', Trans. Amer. Math. Soc. 243 (1978), 251-269.

[21] W. Rudin, Fourier analysis on groups, (Interscience Tracts in Pure and Appl. Math., no. 12, Interscience, New York, 1962).

[22] R. C. Vrem, 'Lacunarity on compact hypergroups', Math. Z. 164 (1978), 93-104.

[23] R. C. Vrem, 'Continuous measures and lacunarity on hypergroups', Trans. Amer. Math. Soc. 269 (1982), 549-556.

[24] D. C. Wilson, 'Lacunarity for tall compact groups: Figà-Talamanca-Rider sets', Monatsh. Math. 101 (1986), 67-74.

\section{Humboldt State University \\ Arcata, California 95521 \\ U.S.A.}

